\section{Use of Angle-Independent M-Mode Sonography for Assessment of Diaphragm Displacement}

To the Editor: We read with interest the article by Orde et $\mathrm{al}^{1}$ describing an angle-independent M-mode sonographic method for assessing diaphragm displacement. We noticed that Orde et $\mathrm{al}^{1}$ showed results that standard M-mode estimation of diaphragmatic motion estimated greater displacements than the angleindependent method. We found a very similar result when we used 2-dimensional speckle tracking to estimate diaphragmatic motion compared to standard Mmode. $^{2}$

We believe that the cause of this systematic overestimation is due to a projection phenomenon, in which the true representation of the diaphragmatic motion is projected onto the M-mode line. The true displacement, in this case, is in the true cranial-caudal direction, and this displacement is multiplied by the $\sec \theta$, where $\theta$ is the angle between the vector defining the cranial-caudal direction and the vector defining the M-mode direction (Figure 1). Since $\sec \theta \geq 1 \quad(0 \leq \theta \leq \pi / 2)$, the length of this projection is at least equal to but generally larger than the more correct displacement represented

Figure 1. Drawing showing several representative positions of the diaphragm (dashed curved line) as it moves from cranial to caudal (left to right) with a final position (solid curved line). The M-mode path is represented by the solid gray arrow, and it is overlaid by a 2-headed hollow arrow representing the apparent excursion of the diaphragm based on the M-mode. The second solid 2-headed arrow marks the cranial-caudal displacement of a specific diaphragmatic point shown as "o." This arrow is more representative of the true displacement. $\theta$ represents the angle between the cranial-caudal direction and the $\mathrm{M}$ mode path.

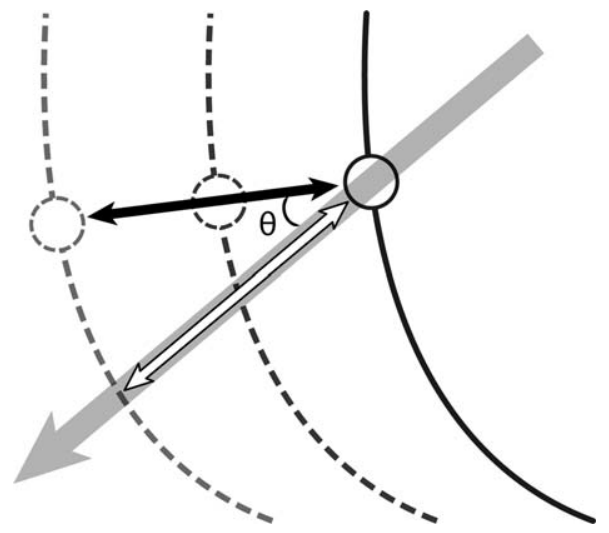

by either the angle-independent M-mode or the 2dimensional speckle-tracking displacement of the diaphragm echo.

The M-mode produces this apparently larger displacement because it is not looking at a single unique point on the diaphragm surface but is actually looking at the continuous intersection of the diaphragm and the M-mode beam as it passes across the direction of the M-mode. This process corresponds to the projection defined above and makes the displacement look greater than it actually is.

\author{
Stephen A. Goutman, MD, MS, \\ James D. Hamilton, PhD, \\ Jonathan M. Rubin, MD, PhD \\ Department of Neurology (S.A.G.) \\ Department of Radiology (J.M.R.) \\ University of Michigan Medical School \\ Ann Arbor, Michigan USA \\ Emerge Inc \\ Marina del Rey, California USA (J.D.H.)
}

doi: 10.1002/jum.14204

\section{References}

1. Orde SR, Boon AJ, Firth DG, Villarraga HR, Sekiguichi H. Use of angle-independent M-mode sonography for assessment of diaphragm displacement. J Ultrasound Med 2016; 35:2615-2621.

2. Goutman SA, Hamilton JD, Swihart B, Foerster B, Feldman EL, Rubin JM. Speckle tracking as a method to measure hemidiaphragm excursion. Muscle Nerve 2017; 55:125-127.

\section{Reply}

To the Editor: We appreciate the insightful comments made by Dr Goutman and his colleagues. ${ }^{1}$ We described traditional "angle-dependent" M-mode as overestimating diaphragm caudal displacement through an "orientation" error. ${ }^{2}$ This concept is identical to the "projection phenomenon" Dr Goutman and his colleagues described, and rather elegantly displayed in Figure 1 of their recent article. ${ }^{3}$ The use of angle sec $\theta$ as a way to describe the difference between angle-dependent M-mode and true diaphragm displacement correctly highlights the limitation of traditional M-mode. ${ }^{3}$ In addition to this projection phenomenon or orientation error, it is important to note that traditional $\mathrm{M}$-mode is prone to translation error, in which the adjacent closer portion of the 